\title{
Design, synthesis, pharmacological evaluation, and docking study of new acridone-based 1,2,4-oxadiazoles as potential anticonvulsant agents
}

Maryam Mohammadi-Khanaposhtani ${ }^{\text {a }}$, Mohammad Shabani ${ }^{\text {b }}$, Mehrdad Faizi ${ }^{\text {c }}$, Iraj Aghaei ${ }^{\text {d }}$ Reza Jahani c, Zainab Sharafi e, Narges Shamsaei Zafarghandi a, Mohammad Mahdavi a, Tahmineh Akbarzadeh $^{\mathrm{a}, \mathrm{f}}$, Saeed Emami ${ }^{\mathrm{g}}$, Abbas Shafiee ${ }^{\mathrm{a}}$, Alireza Foroumadi ${ }^{\mathrm{a}, \mathrm{h}}{ }_{*}$

a Department of Medicinal Chemistry, Faculty of Pharmacy and Pharmaceutical Sciences Research Center, Tehran University of Medical Sciences, Tehran, Iran

${ }^{b}$ Neuroscience Research Center, Institute of Neuropharmacology, Kerman University of Medical Sciences, Kerman, Iran

${ }^{c}$ Department of Pharmacology and Toxicology, School of Pharmacy, Shahid Beheshti University of Medical Sciences, Tehran, Iran

${ }^{d}$ Guilan Road Trauma Research Center, Poursina Hospital, Guilan University of Medical Science, Rasht, Iran

e Department of Pharmaceutical Biotechnology, School of Pharmacy, Shahid Beheshti University of Medical Sciences, Tehran, Iran

${ }^{f}$ Persian Medicine and Pharmacy Research Center, Tehran University of Medical Sciences, Tehran, Iran

${ }^{g}$ Department of Medicinal Chemistry and Pharmaceutical Sciences Research Center, Faculty of Pharmacy, Mazandaran University of Medical Sciences, Sari, Iran

${ }^{h}$ Drug Design and Development Research Center, Tehran University of Medical Sciences, Tehran, Iran

\begin{abstract}
A number of acridone-based oxadiazoles 11a-n have been synthesized and evaluated for their anticonvulsant activity against pentylenetetrazole (PTZ)- and maximal electroshock (MES)-induced seizures in mice. Also, their neurotoxicity was evaluated by the rotarod test. Most of the compounds exhibited better anticonvulsant activity and higher safety respect to the standard drug, phenobarbital. Among the tested derivatives, compounds $11 \mathrm{l}$ with $\mathrm{ED}_{50}$ value of $2.08 \mathrm{mg} / \mathrm{kg}$ was the most potent compound in the PTZ test. The anticonvulsant effect of compound 111 was blocked by flumazenil, suggesting the involvement of benzodiazepine (BZD) receptors in the anticonvulsant activity of prototype compound 111. Also, docking study of compound 111 in the BZD-binding site of $\mathrm{GABA}_{\mathrm{A}}$ receptor confirms possible binding of compound $\mathbf{1 1 1}$ with BZD receptors.
\end{abstract}

Keywords: Anticonvulsant agents; Acridone; Benzodiazepine (BZD) receptors; Docking study; 1,2,4-Oxadiazole

\footnotetext{
* Corresponding author. Tel.: +98 21 66954708; fax: +98 21 66461178; E-mail address: aforoumadi@yahoo.com
} 


\section{Introduction}

Epilepsy is a common neurological disorder that affects approximately 60 million people of the world's population [1]. Although there are many anticonvulsant drugs on the market, the use of most drugs is associated with many side effects and extended drug interactions [2]. On the other hand, anticonvulsant drugs in about $70 \%$ of patients with epilepsy can be effective and the treatment of epilepsy remains still inadequate [3,4]. Thus, the development of more effective and safer antiepileptic drugs is an important goal for many of the medicinal chemists.

Various derivatives bearing 5-membered heterocycles such as oxadiazoles, triazoles, and thiadiazoles have been synthesized and evaluated for their anticonvulsant activities $[5,6]$. Particularly, Lankau et al. reported the anticonvulsant activity of 3-aryl-1,2,4-oxadiazole I (Fig. 1) in a variety of experimental models. They found that these compounds act as selective GABA potentiating compounds with no interaction to the benzodiazepine (BZD) binding site [6]. On the other hand, 10-(2-dimethylaminopropyl)-9-acridinone II (Fig. 1) has been reported as a potent convulsant agent [7]. In continuation of our efforts to find new bioactive acridones [8], we have designed a series of acridone-based 1,2,4-oxadiazoles 11a-n (Fig. 1) as new anticonvulsant agents. Presumably, the designed compounds can act as anticonvulsant agents (such as diazepam and estazolam) through benzodiazepine (BZD) receptors; they have the requirements for binding to these receptors: an aromatic ring (A), a coplanar proton-accepting group in a suitable distance (B), and second out-of-plane aromatic ring (C) that the last case could potentiate binding to the receptor especially for agonists (Fig. 2) [9]. Thus we report here, the synthesis, pharmacological evaluation, and docking study of 10-(3-aryl-[1,2,4]oxadiazol-5-ylmethyl)-10H-acridin-9-one derivatives 11a-n as potential anticonvulsant agents. 


\section{Chemistry}

The designed compounds 11a-n were synthesized according to Scheme 1. The synthetic pathway was started from the Ullmann condensation reaction of 2-bromobenzoic acid $\mathbf{1}$ and different anilines 2 using potassium carbonate and copper in refluxing EtOH, to afford 2-arylamino benzoic acids 3 [10]. Compounds $\mathbf{3}$ easily participated in the cyclization reaction in the presence of PPA at $100{ }^{\circ} \mathrm{C}$ and afforded acridones 4 [11]. On the other hand, 3-aryl-5-(chloromethyl)1,2,4-oxadiazole derivatives $\mathbf{1 0}$ were prepared starting from appropriate benzonitriles $\mathbf{5}$ [12]. Accordingly, benzonitrile derivatives $\mathbf{5}$ and hydroxylamine hydrochloride (6) reacted in refluxing $\mathrm{EtOH}$ in the presence of sodium hydroxide to obtain hydroxybenzamidines 7 . The reaction of compounds 7 and chloroacetyl chloride $(\mathbf{8})$ in the presence of potassium carbonate in dry acetone gave $N^{\prime}$-(2-chloroacetoxy)-4-substituted benzimidamide 9 which underwent the further cyclization reaction in refluxing toluene to give 3-aryl-5-(chloromethyl)-1,2,4-oxadiazole derivatives 10. Finally, the acridone derivatives $\mathbf{4}$ were reacted with 1,2,4-oxadiazole derivatives 10 in the presence of potassium tert-butoxide in DMSO at room temperature to afford the corresponding products $\mathbf{1 1}$ in good yields (55-70\%).

\section{Results and discussion}

\subsection{Anticonvulsant activity}

All the synthesized compounds 11a-n were screened for their anticonvulsant activity by using pentylenetetrazole (PTZ) and maximal electroshock (MES) tests in mice [13]. The results were compared with phenobarbital and diazepam as standard anticonvulsant drugs. Structurally, the designed compounds 11a-n could be categorized into three groups considering the type of 
substituent on the attached phenyl on 3-position of 1,2,4-oxadiazole ring: 4-methyl, 4-chloro, and 4-methoxy derivatives (11a-f, 11g-k and 11l-n). In each series, substituents on the 2-position of acridone ring were altered to optimize the anticonvulsant activity (Table 1).

\subsubsection{Anticonvulsant activity against PTZ-induced seizures}

As can be seen in Table 1, all compounds with exception of 11d and 11e had potent anticonvulsant activity in PTZ-induced seizures test, comparable or more potent than the standard drug phenobarbital. Among the tested compounds, 4-methoxyphenyl derivatives 111 and $11 \mathrm{~m}$ with $\mathrm{ED}_{50}$ values of $\approx 2.1 \mathrm{mg} / \mathrm{kg}$ were the most potent compounds against PTZ-induced seizures. Although these compounds were more active than phenobarbital, but their activity was less than that of diazepam.

In the 4-methyl derivatives 11a-f $\left(R_{2}=M e\right)$, compounds 11a-c showed almost same anticonvulsant activities $\left(\mathrm{ED}_{50}\right.$ values of $\approx 6.3 \mathrm{mg} / \mathrm{kg}$ ). The comparison of 2-substituted acridones 11b-f with unsubstituted derivative $11 a\left(R_{1}=H\right)$ revealed that the chloro or methoxy substituents were tolerated, while the bromo, methyl and ethyl substituents diminished the activity. The obtained $\mathrm{ED}_{50}$ values of compounds $11 \mathrm{~g}-\mathbf{k}\left(\mathrm{R}_{2}=\mathrm{Cl}\right)$ were less than those of their corresponding 4-methyl analogs 11a-e $\left(\mathrm{R}_{2}=\mathrm{Me}\right)$. Thus, the replacement of methyl with chlorine group at $\mathrm{R}_{2}$ increases the anticonvulsant activity. In the 4-chloro series $\mathbf{1 1 g - k}\left(\mathrm{R}_{2}=\mathrm{Cl}\right)$, better results were obtained with 2-chloro and 2-methoxy substitution on the acridone ring. The obtained results for limited series of 4-methoxy derivatives 11l-n revealed that the 4-methoxy substituent on the phenyl-oxadiazole moiety improved the anticonvulsant activity. Notably, 4-methoxyphenyloxadiazole derivatives $\mathbf{1 1 l}$ and $\mathbf{1 1 m}$ bearing a $2-\mathrm{Cl}$ or $2-\mathrm{MeO}$ on the acridone ring showed the highest activity among the all series. 


\subsubsection{Anticonvulsant activity against MES-induced seizures}

The obtained results from MES test revealed that all compounds had potent anticonvulsant activity $\left(\mathrm{ED}_{50} \mathrm{~s}=2.24-8.71 \mathrm{mg} / \mathrm{kg}\right)$. Compound $11 \mathrm{~b}$ with $\mathrm{ED}_{50}$ value of $2.24 \mathrm{mg} / \mathrm{kg}$ found to be the most potent derivative against MES-induced seizures in mice. Furthermore, compounds 11a, 11e, 11h, 11j and $11 \mathrm{~m}$ displayed $\mathrm{ED}_{50}$ values $\leq 2.87 \mathrm{mg} / \mathrm{kg}$, being slightly less potent than compound 11b. While all compounds were more active than phenobarbital, but their activity was less than that of diazepam. The comparison of obtained results from PTZ and MES tests revealed that most compounds had lower $\mathrm{ED}_{50} \mathrm{~s}$ in the MES test respect to PTZ model of epilepsy. Even compounds 11d and 11e which were inactive in the PTZ test, showed significant activity against MES-induced seizures. The alteration of substituent on the acridone ring and on the 3-phenyl1,2,4-oxadiazole moiety ( $\mathrm{R}_{1}$ and $\mathrm{R}_{2}$, respectively) had slight effect on the potency of compounds against MES-induced seizures.

\subsection{Neurotoxicity}

All compounds were screened for their neurotoxicity in mice by using rotarod test. The obtained results were listed in Table 1 as $\mathrm{TD}_{50}$ values $(\mathrm{mg} / \mathrm{kg})$. As seen from data, all compounds displayed neurotoxicity at very high doses $\left(\mathrm{TD}_{50} \mathrm{~S} \geq 438.3 \mathrm{mg} / \mathrm{kg}\right)$. The $\mathrm{TD}_{50}$ value for the standard drug phenobarbital was $68.9 \mathrm{mg} / \mathrm{kg}$ being significantly less than those of title compounds. Based on the obtained results from PTZ, MES and rotarod tests, the protective index

$\left(\mathrm{PI}=\mathrm{TD}_{50} / \mathrm{ED}_{50}\right)$ was calculated for all compounds and presented in Table 1 . The PI values of compounds revealed that the designed compounds enable to display good anticonvulsant activity with high safety profile.

\subsection{Study on possible mechanism of action}


To gain insight into the mechanism of action of the most potent compound 11l, flumazenil test were performed. In PTZ test, flumazenil, a well-known benzodiazepine antagonist, antagonized anticonvulsant effects of active compound 11, which confirms the involvement of BZD receptors in the anticonvulsant activity of prototype compound $\mathbf{1 1 1}$.

\subsection{Docking study}

To confirm whether the anticonvulsant activity of the synthesized compounds mediates through $\mathrm{BZD}$ receptors, the docking of active compound $\mathbf{1 1 1}$ in the $\mathrm{BZD}$-binding site of $\mathrm{GABA}_{\mathrm{A}}$ receptor $(\alpha 1 \beta 2 \gamma 2)$ examined by Autodock Tools (1.5.6). Interactions of the diazepam and target compound $\mathbf{1 1 l}$ with amino acids of BZD-binding pocket of $\mathrm{GABA}_{\mathrm{A}}$ receptor were constructed by Discovery Studio 4.0 Client and showed in Figures 3a and 3b, respectively. As can be seen in Fig. 3a, the most important residues in the binding mode of diazepam are $\alpha 1$ Thr206, $\alpha 1$ Tyr 209, $\alpha 1$ His 101, $\alpha 1$ Tyr159, and $\gamma 2$ Phe77 [14]. The acridone ring of compound 111 is responsible for two important interactions with BZD-binding pocket of $\mathrm{GABA}_{\mathrm{A}}$ : $\pi$-cation interaction with the positively charged nitrogen of $\alpha 1$ His101 and $\pi-\pi$ interaction whit $\gamma 2$ Phe77 (Fig. 3b). It is obvious that there is an hydrogen bond between N-2 of 1,2,4-oxadiazole ring and $\alpha 1 \mathrm{Thr} 206$. Also, $\alpha 1$ Tyr209 possessing aromatic moiety is involved in a $\pi-\pi$ interaction whit 3 -phenyl moiety. Therefore, the results showed that binding mode of compound 111 in the BZD-binding pocket of $\mathrm{GABA}_{\mathrm{A}}$ receptor resembled to that of diazepam.

\subsection{Cytotoxicity}

Potentially, the acridone/acridine derivatives might intercalate with the double-stranded DNA structure thus inducing toxic effects [15]. So we studied the potential cytotoxic effects of this class of compounds. All derivatives 11a-n were evaluated for their cytotoxicity against MCF-7, 
T-47D, and MDA-MB-231 cell lines. The tested compounds showed no in vitro cytotoxic activity against cancer cell lines $\left(\mathrm{IC}_{50}\right.$ values $\left.>50 \mu \mathrm{M}\right)$. The obtained $\mathrm{IC}_{50} \mathrm{~s}$ of the standard drug etoposide were 12.4, 11.8 and 15.7 $\mu \mathrm{M}$ against MCF-7, T-47D, and MDA-MB-231, respectively.

\subsection{Prediction of $B B B$ permeability}

Anticonvulsant agents are CNS-active compounds, so they should have good BBB permeability. Several computational methods have been reported for the prediction of BBB penetration [16]. One of them is the measurement of $\operatorname{logBB}$, which is the ratio of the steady-state concentrations of the compound between the brain and the blood [i.e., $\left.\log \left(\mathrm{C}_{\text {brain }} / \mathrm{C}_{\text {blood }}\right)\right]$. Accordingly, compounds with $\log \mathrm{BB}<-1.0$ will have poor distributed to the brain [17]. Clark has reported an equation for measuring the $\log \mathrm{BB}$ as follow: $\log \mathrm{BB}=-0.0148 \times \mathrm{PSA}+0.152 \times c \log P+0.139$ [18]. The $\log B B$ values of compounds 11a-n were calculated by the equation and presented in Table S1 (Supplementary Material). The measured $\operatorname{logBB}$ values for compounds 11a-n were ranging from -0.688 to 0.249 . As seen from data, compounds $\mathbf{1 1 d}$ and $\mathbf{1 1} \mathbf{i}$ had more favorable $\log \mathrm{BB}$ values. Also, the prototype compound $\mathbf{1 1 1}$ had $\log \mathrm{BB}=0.185$, being acceptable value for a CNS-active compound.

\section{Conclusion}

We designed and synthesized a series of $10 \mathrm{H}$-acridin-9-one derivatives bearing 10-(3-aryl$[1,2,4]$ oxadiazol-5-ylmethyl)- moiety as novel anticonvulsant agents. Most of compounds showed better anticonvulsant activities in comparison to phenobarbital as the standard drug. The 2-chloro-acridinone derivative $\mathbf{1 1 1}$ was the most potent compound in the PTZ test $\left(\mathrm{ED}_{50}=2.08\right.$ $\mathrm{mg} / \mathrm{kg}$ ). Furthermore, compounds 11a, 11b, 11e, 11h, 11j, and 11m with $\mathrm{ED}_{50}$ values $\leq 2.87$ 
$\mathrm{mg} / \mathrm{kg}$ were more promising compounds in the MES test, being at least 7 times more potent than phenobarbital. The safety profile of all compounds was better than that of phenobarbital. The experimental investigation of mechanism with flumazenil test and in silico studies by using molecular docking simulations showed that the prototype compound $\mathbf{1 1}$ can act as BZD-receptor agonist.

\section{Experimental}

Melting points were measured with a Kofler hot stage apparatus and are uncorrected. ${ }^{1} \mathrm{H}$ and ${ }^{13} \mathrm{C}$ NMR spectra were recorded by a Bruker FT-500, using TMS as an internal standard. IR spectra were obtained by a Nicolet Magna FTIR 550 spectrophotometer (KBr disks). MS were recorded with an Agilent Technology (HP) mass spectrometer operating at an ionization potential of 70 eV. Elemental analysis was performed with an Elementar Analysensystem GmbH VarioEL CHNS mode.

\subsection{General procedure for the synthesis of 2-arylaminobenzoic acids $\mathbf{3}$}

A solution of 2-bromobenzoic acid $(\mathbf{1}, 8.5 \mathrm{mmol})$, aniline derivative 2 (159.0 $\mathrm{mmol})$, copper powder $(4.7 \mathrm{mmol})$, and potassium carbonate $(119.0 \mathrm{mmol})$ in ethanol $(80 \mathrm{ml})$ was heated under reflux for $7 \mathrm{~h}$. After completion of reaction (checked by TLC), the reaction mixture was cooled down to room temperature, poured into hot water and boiled in the presence of activated charcoal for 15 min. Then, it was filtered through celite and the filtrate was acidified with $\mathrm{HCl}$ to obtain precipitated product which was purified using recrystallization from ethanol to obtain pure products $(80-85 \%)$.

\subsection{General procedure for the synthesis of acridone derivatives 4}


A mixture of appropriate 2-arylaminobenzoic acid $3(1 \mathrm{mmol})$ and polyphosphoric acid (10 mmol) was heated at $100{ }^{\circ} \mathrm{C}$ for $3 \mathrm{~h}$. Upon completion, the reaction mixture was poured into hot water $(50 \mathrm{ml})$. After cooling to room temperature, the mixture was made alkaline by ammonia solution. The obtained yellow precipitates were filtered off, washed with hot water, and purified using recrystallization from acetic acid to obtain the pure products $(80-85 \%)$.

\subsection{General procedure for the synthesis of 3-aryl-5-(chloromethyl)-1,2,4-oxadiazole derivatives}

\section{0}

An aqueous solution of sodium hydroxide $(20 \mathrm{mmol}$ in $10 \mathrm{ml})$ was added to a solution of benzonitrile 5 (10 mmol) and hydroxylamine hydrochloride $6(20 \mathrm{mmol})$ in EtOH $(50 \mathrm{ml})$ in a dropwise manner at room temperature. Then, the mixture was heated under reflux for $18 \mathrm{~h}$. After completion of the reaction (checked by TLC), the solvent was evaporated under reduced pressure, water $(50 \mathrm{ml})$ was added to the residue, and the $\mathrm{pH}$ of solution was set at $\mathrm{pH}=2$ using $\mathrm{HCl}(1 \mathrm{~N})$. The mixture was extracted using ethyl acetate $(2 \times 25 \mathrm{ml})$. The aqueous solution was cooled to $0{ }^{\circ} \mathrm{C}$ and neutralized using sodium carbonate solution. The obtained precipitate was filtered off, washed with water, and dried at $60{ }^{\circ} \mathrm{C}$ to obtain compound 7. In the next step, chloroacetyl chloride $\mathbf{8}(12 \mathrm{mmol})$ was added to a stirring solution of compound $\mathbf{7}$ (11.2 mmol) and $\mathrm{K}_{2} \mathrm{CO}_{3}(11 \mathrm{mmol})$ in dry acetone $(25 \mathrm{ml})$ in a dropwise manner during $10 \mathrm{~min}$. The reaction mixture was stirred at room temperature for $24 \mathrm{~h}$. After reaction completion, the solvent was evaporated under reduced pressure, the obtained white precipitate was dissolved in ethyl acetate $(50 \mathrm{ml})$, and the organic phase was washed with water $(4 \times 20 \mathrm{ml})$ and brine $(20 \mathrm{ml})$. The organic phase was dried over $\mathrm{Na}_{2} \mathrm{SO}_{4}$ and the solvent was evaporated under reduced pressure. The residue was recrystallized from EtOH to obtain pure $N^{\prime}$-(2-chloroacetoxy)-benzimidamide 9. A suspension of compound $9(8 \mathrm{mmol})$ in toluene $(50 \mathrm{ml})$ was heated under reflux for $5 \mathrm{~h}$. Then, 
the solvent was evaporated under reduced pressure and the obtained yellow oily residue was recrystallized from petroleum ether to give pure yellow 3-aryl-5-(chloromethyl)-1,2,4-oxadiazole derivative $10(65-70 \%)$.

5.4. General procedure for the synthesis of 10-(3-aryl-[1,2,4]oxadiazol-5-ylmethyl)-10Hacridin-9-one derivatives 11a-n

A suspension of acridone derivative $4(0.5 \mathrm{mmol})$ and potassium tert-butoxide $(0.07 \mathrm{mmol})$ in DMSO $(0.5 \mathrm{ml})$ was stirred at room temperature for $30 \mathrm{~min}$. Then, the mixture was added to a solution of 3-aryl-5-(chloromethyl)-1,2,4-oxadiazole derivative 10 (1 mmol) in DMSO (2 ml) and the reaction mixture was stirred at room temperature for $3 \mathrm{~h}$. After that, it was poured into crushed ice and the aqueous solution was extracted with dichloromethane $(3 \times 10 \mathrm{ml})$, washed with water, and dried over $\mathrm{Na}_{2} \mathrm{SO}_{4}$. The solvent was evaporated under reduced pressure and the residue was purified by flash chromatography on silica gel eluting with petroleum ether/ethyl acetate (9:1) to obtain pure products $(55-70 \%)$.

5.4.1. 10-(3-p-Tolyl-[1,2,4]oxadiazol-5-ylmethyl)-10H-acridin-9-one (11a). White crystals; yield: $76 \%$, mp 239-241 ${ }^{\circ} \mathrm{C}$. IR (KBr): 3069, 2904, 1647, 1604, 1486, $1460 \mathrm{~cm}^{-1} .{ }^{1} \mathrm{H}$ NMR (400 MHz, DMSO-d $)_{6}: 8.39\left(\mathrm{~d}, J=8.0 \mathrm{~Hz}, 2 \mathrm{H}, \mathrm{H}_{1}, \mathrm{H}_{8}\right), 7.84\left(\mathrm{~m}, 4 \mathrm{H}, \mathrm{H}_{3}, \mathrm{H}_{4}, \mathrm{H}_{5}, \mathrm{H}_{6}\right), 7.79(\mathrm{~d}, J=8.2$ $\left.\mathrm{Hz}, 2 \mathrm{H}, \mathrm{H}_{2^{\prime}}, \mathrm{H}_{6^{\prime}}\right), 7.42-7.38\left(\mathrm{~m}, 2 \mathrm{H}, \mathrm{H}_{2}, \mathrm{H}_{7}\right), 7.31\left(\mathrm{~d}, J=8.2 \mathrm{~Hz}, 2 \mathrm{H}, \mathrm{H}_{3^{\prime}}, \mathrm{H}_{5^{\prime}}\right), 6.25\left(\mathrm{~s}, 2 \mathrm{H}, \mathrm{CH}_{2}\right)$, $2.34\left(\mathrm{~s}, 3 \mathrm{H}, \mathrm{CH}_{3}\right) .{ }^{13} \mathrm{C} \mathrm{NMR}\left(100 \mathrm{MHz}, \mathrm{DMSO}-d_{6}\right): 177.2,176.4,168.3,142.3,142.2,135.0$, 127.5, 127.3, 123.3, 122.6, 122.3, 116.4, 42.9, 21.5. MS m/z (\%) $367\left(\mathrm{M}^{+}, 70\right), 253(20), 208$ (100), 180 (31), 125 (99), 91 (33), 65 (9), 43 (8). Anal. Calcd for $\mathrm{C}_{23} \mathrm{H}_{17} \mathrm{~N}_{3} \mathrm{O}_{2}$ : C, 75.19; H, 4.66; N, 11.44. Found: C, 75.34; H, 4.21; N, 11.68. 
5.4.2. 2-Chloro-10-(3-p-tolyl-[1,2,4]oxadiazol-5-ylmethyl)-10H-acridin-9-one (11b). White crystals; yield: $69 \%, \mathrm{mp}>250{ }^{\circ} \mathrm{C}$. IR (KBr): $3093,2919,1635,1597,1487,1457 \mathrm{~cm}^{-1} .{ }^{1} \mathrm{H}$ NMR $\left(500 \mathrm{MHz}, \mathrm{CDCl}_{3}\right): 8.61\left(\mathrm{dd}, J=8.1,1.4 \mathrm{~Hz}, 1 \mathrm{H}, \mathrm{H}_{8}\right), 8.57\left(\mathrm{~d}, J=2.5 \mathrm{~Hz}, 1 \mathrm{H}, \mathrm{H}_{1}\right), 7.96(\mathrm{~d}, J=$ $\left.8.1 \mathrm{~Hz}, 2 \mathrm{H}, \mathrm{H}_{4}, \mathrm{H}_{5}\right), 7.82\left(\mathrm{td}, J=7.8,1.5 \mathrm{~Hz}, 1 \mathrm{H}, \mathrm{H}_{3}\right), 7.73\left(\mathrm{dd}, J=9,2.5 \mathrm{~Hz}, 1 \mathrm{H}, \mathrm{H}_{6}\right), 7.65(\mathrm{~d}, J$ $\left.=8.2 \mathrm{~Hz}, 2 \mathrm{H}, \mathrm{H}_{2^{\prime}}, \mathrm{H}_{6^{\prime}}\right), 7.41\left(\mathrm{t}, J=7.5 \mathrm{~Hz}, 1 \mathrm{H}, \mathrm{H}_{7}\right), 7.31\left(\mathrm{~d}, J=8.2 \mathrm{~Hz}, 2 \mathrm{H}, \mathrm{H}_{3^{\prime}}, \mathrm{H}_{5^{\prime}}\right), 5.79(\mathrm{~s}, 2 \mathrm{H}$, $\mathrm{CH}_{2}$ ), 2.45 (s, 3H, $\left.\mathrm{CH}_{3}\right) .{ }^{13} \mathrm{C} \mathrm{NMR}\left(125 \mathrm{MHz}, \mathrm{CDCl}_{3}\right)$ : 175.6, 174.1, 167.5, 142.1, 141.0, 138.4, $135.0,134.3,132.2,128.6,128.3,127.1,126.6,125.7,124.0,123.0,122.0,119.6,117.0,42.8$, 21.0. MS m/z (\%) $403\left(\left[\mathrm{M}^{+}+2\right], 22\right), 401\left(\mathrm{M}^{+}, 68\right), 242$ (100), 228 (24), 214 (29), 200 (26), 179 (21), 152 (25), 91 (20), 63 (10), 43 (9). Anal. Calcd for $\mathrm{C}_{23} \mathrm{H}_{16} \mathrm{ClN}_{3} \mathrm{O}_{2}$ : C, 68.74; H, 4.01; N, 10.46. Found: C, 68.39; H, 3.94; N, 10.32 .

5.4.3. 2-Methoxy-10-(3-p-tolyl-[1,2,4]oxadiazol-5-ylmethyl)-10H-acridin-9-one (11c). Yellow crystals; yield: $73 \%$, mp $226-228{ }^{\circ} \mathrm{C}$. IR (KBr): 3064, 2922, 1616, 1591, 1488, $1461 \mathrm{~cm}^{-1} .{ }^{1} \mathrm{H}$ $\operatorname{NMR}\left(500 \mathrm{MHz}, \mathrm{CDCl}_{3}\right): 8.63\left(\mathrm{~d}, J=7.9 \mathrm{~Hz}, 1 \mathrm{H}, \mathrm{H}_{8}\right), 8.03\left(\mathrm{~d}, J=2.2 \mathrm{~Hz}, 1 \mathrm{H}, \mathrm{H}_{1}\right), 7.97(\mathrm{~d}, J=$ $\left.7.8 \mathrm{~Hz}, 2 \mathrm{H}, \mathrm{H}_{2}, \mathrm{H}_{6^{\prime}}\right), 7.79\left(\mathrm{t}, J=7.8 \mathrm{~Hz}, 1 \mathrm{H}, \mathrm{H}_{3}\right), 7.66\left(\mathrm{t}, J=8.6 \mathrm{~Hz}, 2 \mathrm{H}, \mathrm{H}_{4}, \mathrm{H}_{5}\right), 7.43(\mathrm{dd}, J=$ 7.9, $\left.2.2 \mathrm{~Hz}, 1 \mathrm{H}, \mathrm{H}_{6}\right), 7.39\left(\mathrm{t}, J=7.4 \mathrm{~Hz}, 1 \mathrm{H}, \mathrm{H}_{7}\right), 7.31\left(\mathrm{~d}, J=7.8 \mathrm{~Hz}, 2 \mathrm{H}, \mathrm{H}_{3^{\prime}}, \mathrm{H}_{5^{\prime}}\right), 5.81(\mathrm{~s}, 2 \mathrm{H}$, $\mathrm{CH}_{2}$ ), 3.9 (s, 3H, $\mathrm{OCH}_{3}$ ), 2.38 (s, 3H, $\left.\mathrm{CH}_{3}\right) .{ }^{13} \mathrm{C} \mathrm{NMR} \mathrm{(125} \mathrm{MHz,} \mathrm{CDCl}_{3}$ ): 176.5, 175.9, 167.7, 154.6, 143.3, 141.9, 137.0, 136.9, 134.6, 128.6, 128.3, 127.1, 124.4, 123.9, 121.6, 120.4, 118.2, 116.6, 106.6, 55.9, 42.9, 20.8. Anal. Calcd for $\mathrm{C}_{24} \mathrm{H}_{19} \mathrm{~N}_{3} \mathrm{O}_{3}$ : C, 72.53; H, 4.82; N, 10.57. Found: C, 72.41; H, 5.03; N, 10.34 .

5.4.4. 2-Bromo-10-(3-p-tolyl-[1,2,4]oxadiazol-5-ylmethyl)-10H-acridin-9-one (11d). White crystals; yield: $71 \%, \mathrm{mp}>250{ }^{\circ} \mathrm{C}$. IR (KBr): $3111,2923,1635,1598,1486,1463 \mathrm{~cm}^{-1} .{ }^{1} \mathrm{H}$ NMR $\left(500 \mathrm{MHz}, \mathrm{CDCl}_{3}\right): 8.72\left(\mathrm{~d}, J=2.4 \mathrm{~Hz}, 1 \mathrm{H}, \mathrm{H}_{1}\right), 8.60\left(\mathrm{dd}, J=8,1.3 \mathrm{~Hz}, 1 \mathrm{H}, \mathrm{H}_{8}\right), 7.96(\mathrm{~d}, J=8.1$ $\left.\mathrm{Hz}, 2 \mathrm{H}, \mathrm{H}_{4}, \mathrm{H}_{5}\right), 7.81\left(\mathrm{dd}, J=9,2.4 \mathrm{~Hz}, 1 \mathrm{H}, \mathrm{H}_{6}\right), 7.82\left(\mathrm{td}, J=7.9,1.4 \mathrm{~Hz}, 1 \mathrm{H}, \mathrm{H}_{3}\right), 7.65(\mathrm{~d}, J=$ 
$\left.8.6 \mathrm{~Hz}, 2 \mathrm{H}, \mathrm{H}_{2}, \mathrm{H}_{6^{\prime}}\right), 7.42\left(\mathrm{t}, J=7.5 \mathrm{~Hz}, 1 \mathrm{H}, \mathrm{H}_{7}\right), 7.31\left(\mathrm{~d}, J=8.6 \mathrm{~Hz}, 2 \mathrm{H}, \mathrm{H}_{3^{\prime}}, \mathrm{H}_{5^{\prime}}\right), 5.82(\mathrm{~s}, 2 \mathrm{H}$, $\mathrm{CH}_{2}$ ), 2.44 (s, 3H, $\left.\mathrm{CH}_{3}\right) .{ }^{13} \mathrm{C}$ NMR (125 MHz, $\mathrm{CDCl}_{3}$ ): 176.0, 174.7, 165.2, 142.9, 142.1, 141.3, $137.2,136.7,135.4,128.9,128.6,128.3,127.1,124.0,123.5,121.1,120.0,117.0,114.4,42.9$, 20.3. Anal. Calcd for $\mathrm{C}_{23} \mathrm{H}_{16} \mathrm{BrN}_{3} \mathrm{O}_{2}: \mathrm{C}, 61.90 ; \mathrm{H}, 3.61 ; \mathrm{N}, 9.42$. Found: $\mathrm{C}, 61.71 ; \mathrm{H}, 3.83 ; \mathrm{N}$, 9.63 .

5.4.5. 2-Methyl-10-(3-p-tolyl-[1,2,4]oxadiazol-5-ylmethyl)-10H-acridin-9-one (11e). White crystals; yield: $73 \%, \mathrm{mp}>250{ }^{\circ} \mathrm{C}$. IR (KBr): 3078, 2919, 1638, 1596, 1483, $1457 \mathrm{~cm}^{-1} .{ }^{1} \mathrm{H}$ NMR $\left(500 \mathrm{MHz}, \mathrm{CDCl}_{3}\right): 8.63\left(\mathrm{~d}, J=8.0 \mathrm{~Hz}, 1 \mathrm{H}, \mathrm{H}_{8}\right), 8.42\left(\mathrm{~s}, 1 \mathrm{H}, \mathrm{H}_{1}\right), 7.97\left(\mathrm{~d}, J=8.0 \mathrm{~Hz}, 2 \mathrm{H}, \mathrm{H}_{4}\right.$, $\left.\mathrm{H}_{5}\right), 7.80-7.77\left(\mathrm{~m}, 1 \mathrm{H}, \mathrm{H}_{3}\right), 7.64-7.61\left(\mathrm{~m}, 1 \mathrm{H}, \mathrm{H}_{6}\right), 7.56\left(\mathrm{~d}, J=8.7 \mathrm{~Hz}, 2 \mathrm{H}, \mathrm{H}_{2}, \mathrm{H}_{6}\right), 7.38(\mathrm{t}, J=$ $\left.7.4 \mathrm{~Hz}, 1 \mathrm{H}, \mathrm{H}_{7}\right), 7.31\left(\mathrm{~d}, \mathrm{~J}=8.7 \mathrm{~Hz}, 2 \mathrm{H}, \mathrm{H}_{3^{\prime}}, \mathrm{H}_{5^{\prime}}\right), 5.80\left(\mathrm{~s}, 2 \mathrm{H}, \mathrm{CH}_{2}\right), 2.52\left(\mathrm{~s}, 3 \mathrm{H}, \mathrm{CH}_{3}\right), 2.44(\mathrm{~s}$, $\left.3 \mathrm{H}, \mathrm{CH}_{3}\right) .{ }^{13} \mathrm{C} \mathrm{NMR}\left(125 \mathrm{MHz}, \mathrm{CDCl}_{3}\right): 176.6,176.4,166.0,143.3,142.1,140.4,136.4,136.0$, 134.5, 131.2, 129.2, 128.6, 128.3, 127.1, 126.3, 123.9, 121.7, 116.8, 116.6, 43.0, 20.9, 20.6. Anal. Calcd for $\mathrm{C}_{24} \mathrm{H}_{19} \mathrm{~N}_{3} \mathrm{O}_{2}$ : C, 75.57; H, 5.02; N, 11.02. Found: C, 75.72; H, 4.89; N, 11.26 .

5.4.6. 2-Ethyl-10-(3-p-tolyl-[1,2,4]oxadiazol-5-ylmethyl)-10H-acridin-9-one (11f). Yellow crystals; yield: $64 \%, \mathrm{mp}>250{ }^{\circ} \mathrm{C}$. IR (KBr): $3065,2922,1635,1593,1480,1450 \mathrm{~cm}^{-1} .{ }^{1} \mathrm{H}$ NMR $\left(500 \mathrm{MHz}, \mathrm{CDCl}_{3}\right): 8.63\left(\mathrm{dd}, J=7.8,1.4 \mathrm{~Hz}, 1 \mathrm{H}, \mathrm{H}_{8}\right), 8.43\left(\mathrm{~d}, J=1.7 \mathrm{~Hz}, 1 \mathrm{H}, \mathrm{H}_{1}\right), 7.97(\mathrm{~d}, J=$ $\left.8.1 \mathrm{~Hz}, 2 \mathrm{H}, \mathrm{H}_{4}, \mathrm{H}_{5}\right), 7.78\left(\mathrm{~m}, 1 \mathrm{H}, \mathrm{H}_{3}\right), 7.64\left(\mathrm{~m}, 1 \mathrm{H}, \mathrm{H}_{6}\right), 7.58\left(\mathrm{~d}, J=8.7 \mathrm{~Hz}, 2 \mathrm{H}, \mathrm{H}_{2}, \mathrm{H}_{6}\right)^{\prime}, 7.38$ $\left(\mathrm{t}, J=7.4 \mathrm{~Hz}, 1 \mathrm{H}, \mathrm{H}_{7}\right), 7.31\left(\mathrm{~d}, J=8.7 \mathrm{~Hz}, 2 \mathrm{H}, \mathrm{H}_{3^{\prime}}, \mathrm{H}_{5^{\prime}}\right), 5.80\left(\mathrm{~s}, 2 \mathrm{H}, \mathrm{CH}_{2}\right), 2.83(\mathrm{q}, J=7.5 \mathrm{~Hz}$, $\left.2 \mathrm{H}, \mathrm{CH}_{2}\right), 2.44\left(\mathrm{~s}, 3 \mathrm{H}, \mathrm{CH}_{3}\right), 1.35\left(\mathrm{t}, J=7.5 \mathrm{~Hz}, 3 \mathrm{H}, \mathrm{CH}_{3}\right) .{ }^{13} \mathrm{C}$ NMR $\left(125 \mathrm{MHz}, \mathrm{DMSO}-d_{6}\right)$ : $175.5,174.1,167.1,142.7,141.6,140.1,136.9,134.4,133.9,129.5,127.8,126.6,124.5,123.0$, 121.6, 121.2, 116.4, 116.1, 114.0, 55.1, 41.5, 27.3, 15.5. Anal. Calcd for $\mathrm{C}_{25} \mathrm{H}_{21} \mathrm{~N}_{3} \mathrm{O}_{2}$ : C, 75.93; H, 5.35; N, 10.63. Found: C, 75.71; H, 5.53; N, 10.24. 
5.4.7. 10-[3-(4-Chlorophenyl)-[1,2,4]oxadiazol-5-ylmethyl]-10H-acridin-9-one (11g). White crystals; yield: $68 \%$, mp $244-245{ }^{\circ} \mathrm{C}$. IR (KBr): $3114,2922,1646,1603,1488,1464 \mathrm{~cm}^{-1} .{ }^{1} \mathrm{H}$ NMR (500 MHz, CDCl $): 8.64\left(\mathrm{~d}, J=7.9 \mathrm{~Hz}, 2 \mathrm{H}, \mathrm{H}_{1}, \mathrm{H}_{8}\right), 8.03\left(\mathrm{~d}, J=8.4 \mathrm{~Hz}, 2 \mathrm{H}, \mathrm{H}_{4}, \mathrm{H}_{5}\right)$, 7.83-7.80 (m, 2H, $\left.\mathrm{H}_{3}, \mathrm{H}_{6}\right), 7.63\left(\mathrm{~d}, J=8.5 \mathrm{~Hz}, 2 \mathrm{H}, \mathrm{H}_{3^{\prime}}, \mathrm{H}_{5^{\prime}}\right), 7.48\left(\mathrm{~d}, J=8.5 \mathrm{~Hz}, \mathrm{H}_{2^{\prime}}, \mathrm{H}_{6^{\prime}}\right), 5.84(\mathrm{~s}$, $\left.2 \mathrm{H}, \mathrm{CH}_{2}\right) .{ }^{13} \mathrm{C} \mathrm{NMR}\left(125 \mathrm{MHz}, \mathrm{CDCl}_{3}\right): 177.1,174.3,167.2,142.3,141.3,136.4,134.7,133.3$, 129.2, 127.1, 123.0, 122.0, 117.0, 42.9. MS m/z (\%) 389 ([M + 2], 21), $387\left(\mathrm{M}^{+}, 67\right), 208(100)$, 228 (24), 193 (29), 174 (23), 152 (26), 91 (22), 65 (13), 43 (10). Anal. Calcd for $\mathrm{C}_{22} \mathrm{H}_{14} \mathrm{ClN}_{3} \mathrm{O}_{2}$ : C, 68.13; H, 3.64; N, 10.83. Found: C, 67.88; H, 3.91; N, 10.65 .

5.4.8. 2-Chloro-10-((3-(4-chlorophenyl)-1,2,4-oxadiazol-5-yl)methyl)acridin-9(10H)-one (11h). Yellow crystals; yield: $64 \%, \mathrm{mp}>250{ }^{\circ} \mathrm{C}$. IR (KBr): 3058, 2924, 1638, 1600, 1487, $1463 \mathrm{~cm}^{-1}$. ${ }^{1} \mathrm{H}$ NMR (500 MHz, $\left.\mathrm{CDCl}_{3}\right)$ : 8.62-8.58 (m, 2H, $\left.\mathrm{H}_{1}, \mathrm{H}_{8}\right), 8.02\left(\mathrm{~d}, J=8.5 \mathrm{~Hz}, 2 \mathrm{H}, \mathrm{H}_{4}, \mathrm{H}_{5}\right), 7.85-$ $7.81\left(\mathrm{~m}, 1 \mathrm{H}, \mathrm{H}_{3}\right), 7.75\left(\mathrm{dd}, J=9,2.6 \mathrm{~Hz}, 1 \mathrm{H}, \mathrm{H}_{6}\right), 7.62\left(\mathrm{~d}, J=8.6 \mathrm{~Hz}, 2 \mathrm{H}, \mathrm{H}_{3^{\prime}}, \mathrm{H}_{5^{\prime}}\right), 7.49(\mathrm{~d}, J=$ $\left.8.6 \mathrm{~Hz}, \mathrm{H}_{2}, \mathrm{H}_{6}{ }^{\prime}\right), 7.43\left(\mathrm{t}, J=7.5 \mathrm{~Hz}, 1 \mathrm{H}, \mathrm{H}_{7}\right), 5.86\left(\mathrm{~s}, 2 \mathrm{H}, \mathrm{CH}_{2}\right) .{ }^{13} \mathrm{C} \mathrm{NMR}\left(125 \mathrm{MHz}, \mathrm{CDCl}_{3}\right)$ : 176.1, 175.2, 163.6, 143.5, 142.2, 141.0, 138.3, 136.1, 135.3, 134.3, 131.3, 127.1, 126.6, 125.7, 123.1, 122.8, 121.6, 119.9, 116.3, 42.9. MS m/z (\%) $423([\mathrm{M}+2], 24), 421\left(\mathrm{M}^{+}, 68\right), 242(100)$, 213 (26), 210 (27), 191 (25), 183 (20), 152 (25), 91 (18), 65 (11), 43 (9). Anal. Calcd for $\mathrm{C}_{22} \mathrm{H}_{13} \mathrm{Cl}_{2} \mathrm{~N}_{3} \mathrm{O}_{2}$ : C, 62.58; H, 3.10; N, 9.95. Found: C, 62.31; H, 3.42; N, 9.63.

5.4.9. 10-[3-(4-Chlorophenyl)-[1,2,4]oxadiazol-5-ylmethyl]-2-methoxy-10H-acridin-9-one (11i). Yellow crystals; yield: $62 \%$, mp $225-227^{\circ} \mathrm{C}$. IR (KBr): 3086, 2927, 1614, 1592, 1494, $1463 \mathrm{~cm}^{-}$ 1. ${ }^{1} \mathrm{H}$ NMR (500 MHz, $\left.\mathrm{CDCl}_{3}\right): 8.64\left(\mathrm{~d}, J=8.0 \mathrm{~Hz}, 1 \mathrm{H}, \mathrm{H}_{8}\right), 8.04-8.01\left(\mathrm{~m}, 3 \mathrm{H}, \mathrm{H} 1, \mathrm{H}_{4}, \mathrm{H}_{5}\right)$, 7.81-7.78 (m, 1H, $\left.\mathrm{H}_{3}\right), 7.62\left(\mathrm{~d}, J=8.6 \mathrm{~Hz}, 2 \mathrm{H}, \mathrm{H}_{3^{\prime}}, \mathrm{H}_{5^{\prime}}\right), 7.48\left(\mathrm{~d}, J=8.6 \mathrm{~Hz}, 2 \mathrm{H}, \mathrm{H}_{2^{\prime}}, \mathrm{H}_{6^{\prime}}\right), 7.44$ $\left(\mathrm{dd}, J=9.2,3.0 \mathrm{~Hz}, 1 \mathrm{H}, \mathrm{H}_{6}\right), 7.39\left(\mathrm{t}, J=7.5 \mathrm{~Hz}, 1 \mathrm{H}, \mathrm{H}_{7}\right), 5.83\left(\mathrm{~s}, 2 \mathrm{H}, \mathrm{CH}_{2}\right), 3.98\left(\mathrm{~s}, 3 \mathrm{H}, \mathrm{OCH}_{3}\right)$. ${ }^{13} \mathrm{C}$ NMR (125 MHz, $\left.\mathrm{CDCl}_{3}\right): 176.4,173.9,164.5,154.3,143.5,135.5,134.3,131.3,130.0$, 
129.1, 127.2, 124.4, 123.9, 122.9, 121.9, 121.5, 118.8, 106.6, 106.1, 56.3, 42.9. Anal. Calcd for $\mathrm{C}_{23} \mathrm{H}_{16} \mathrm{ClN}_{3} \mathrm{O}_{3}: \mathrm{C}, 66.11 ; \mathrm{H}, 3.86 ; \mathrm{N}, 10.06$. Found: $\mathrm{C}, 65.97 ; \mathrm{H}, 4.03 ; \mathrm{N}, 10.37$.

5.4.10. 2-Bromo-10-[3-(4-chlorophenyl)-[1,2,4]oxadiazol-5-ylmethyl]-10H-acridin-9-one (11j). Yellow crystals; yield: $61 \%$, mp $>250{ }^{\circ} \mathrm{C}$. IR (KBr): 3073, 2923, 1637, 1600, 1486, $1462 \mathrm{~cm}^{-1}$. ${ }^{1} \mathrm{H}$ NMR $\left(500 \mathrm{MHz}, \mathrm{CDCl}_{3}\right): 8.73\left(\mathrm{~d}, J=2.4 \mathrm{~Hz}, 1 \mathrm{H}, \mathrm{H}_{1}\right), 8.62\left(\mathrm{dd}, J=8.0,1.3 \mathrm{~Hz}, 1 \mathrm{H}, \mathrm{H}_{8}\right)$, $8.02\left(\mathrm{~d}, J=8.5 \mathrm{~Hz}, 2 \mathrm{H}, \mathrm{H}_{4}, \mathrm{H}_{5}\right), 7.88\left(\mathrm{dd}, J=9.0,2.5 \mathrm{~Hz}, 1 \mathrm{H}, \mathrm{H}_{6}\right), 7.84-7.81\left(\mathrm{~m}, 1 \mathrm{H}, \mathrm{H}_{3}\right), 7.63$ $\left(\mathrm{d}, J=8.8 \mathrm{~Hz}, 2 \mathrm{H}, \mathrm{H}_{3^{\prime}}, \mathrm{H}_{5^{\prime}}\right), 7.55\left(\mathrm{~d}, J=8.8 \mathrm{~Hz}, 2 \mathrm{H}, \mathrm{H}_{2^{\prime}}, \mathrm{H}_{6^{\prime}}\right), 7.40\left(\mathrm{t}, J=8.1 \mathrm{~Hz}, 1 \mathrm{H}, \mathrm{H}_{7}\right), 5.70$ (s, $\left.2 \mathrm{H}, \mathrm{CH}_{2}\right) .{ }^{13} \mathrm{C}$ NMR $\left(125 \mathrm{MHz}, \mathrm{CDCl}_{3}\right): 177.1,173.3,164.1,143.0,142.2,141.3,138.0$, 136.6, 135.1, 134.2, 129.2, 128.9, 127.2, 124.1, 123.5, 122.5, 119.8, 117.0, 114.4, 42.8. Anal. Calcd for $\mathrm{C}_{22} \mathrm{H}_{13} \mathrm{BrClN}_{3} \mathrm{O}_{2}$ : C, 56.62; H, 2.81; N, 9.00. Found: C, 56.38; H, 3.02; N, 9.24.

5.4.11. 10-[3-(4-Chlorophenyl)-[1,2,4]oxadiazol-5-ylmethyl]-2-methyl-10H-acridin-9-one (11k). White crystals; yield: $68 \%$, mp 247-249 ${ }^{\circ} \mathrm{C}$. IR (KBr): 3110, 2922, 1637, 1597, 1486, $1462 \mathrm{~cm}^{-1}$. ${ }^{1} \mathrm{H}$ NMR (500 MHz, $\left.\mathrm{CDCl}_{3}\right): 8.63\left(\mathrm{~d}, J=7.9 \mathrm{~Hz}, 1 \mathrm{H}, \mathrm{H}_{8}\right), 8.42\left(\mathrm{~s}, 1 \mathrm{H}, \mathrm{H}_{1}\right), 8.02(\mathrm{~d}, J=8.4 \mathrm{~Hz}$, $\left.2 \mathrm{H}, \mathrm{H}_{4}, \mathrm{H}_{5}\right), 7.81-7.77\left(\mathrm{~m}, 1 \mathrm{H}, \mathrm{H}_{3}\right), 7.63-7.60\left(\mathrm{~m}, 1 \mathrm{H}, \mathrm{H}_{6}\right), 7.54\left(\mathrm{~d}, J=8.4 \mathrm{~Hz}, 2 \mathrm{H}, \mathrm{H}_{3^{\prime}}, \mathrm{H}_{5^{\prime}}\right), 7.48$ $\left(\mathrm{d}, J=8.4 \mathrm{~Hz}, 2 \mathrm{H}, \mathrm{H}_{2}, \mathrm{H}_{6^{\prime}}\right), 7.39\left(\mathrm{t}, J=7.4 \mathrm{~Hz}, 1 \mathrm{H}, \mathrm{H}_{7}\right), 5.92\left(\mathrm{~s}, 2 \mathrm{H}, \mathrm{CH}_{2}\right), 2.53\left(\mathrm{~s}, 3 \mathrm{H}, \mathrm{CH}_{3}\right)$. ${ }^{13} \mathrm{C}$ NMR (125 MHz, $\left.\mathrm{CDCl}_{3}\right): 176.0,174.1,164.9,143.4,142.1,140.4,136.0,135.4,134.5$, 133.3, 131.2, 130.2, 129.2, 127.1, 126.3, 124.0, 121.7, 116.8, 116.6, 43.0, 21.0. Anal. Calcd for $\mathrm{C}_{23} \mathrm{H}_{16} \mathrm{ClN}_{3} \mathrm{O}_{2}: \mathrm{C}, 68.74 ; \mathrm{H}, 4.01 ; \mathrm{N}, 10.46$. Found: $\mathrm{C}, 68.48 ; \mathrm{H}, 4.28 ; \mathrm{N}, 10.61$.

5.4.12. 2-Chloro-10-[3-(4-methoxyphenyl)-[1,2,4]oxadiazol-5-ylmethyl]-10H-acridin-9-one (11l). White crystals; yield: 65\%, mp > $250{ }^{\circ} \mathrm{C}$. IR (KBr): 3068, 2924, 1636, 1597, 1485, 1458 $\mathrm{cm}^{-1} .{ }^{1} \mathrm{H}$ NMR $\left(500 \mathrm{MHz}, \mathrm{CDCl}_{3}\right): 8.61-8.57\left(\mathrm{~m}, 2 \mathrm{H}, \mathrm{H}_{1}, \mathrm{H}_{8}\right), 8.01\left(\mathrm{~d}, J=8.8 \mathrm{~Hz}, 2 \mathrm{H}, \mathrm{H}_{4}, \mathrm{H}_{5}\right)$, 7.83-7.80 (m, 1H, $\left.\mathrm{H}_{3}\right), 7.74\left(\mathrm{dd}, J=9.3,2.5 \mathrm{~Hz}, 1 \mathrm{H}, \mathrm{H}_{6}\right), 7.65\left(\mathrm{~d}, J=8.7 \mathrm{~Hz}, 2 \mathrm{H}, \mathrm{H}_{2}, \mathrm{H}_{6}\right), 7.00$ $\left(\mathrm{d}, J=8.7 \mathrm{~Hz}, 2 \mathrm{H}, \mathrm{H}_{3}, \mathrm{H}_{5}\right), 5.79\left(\mathrm{~s}, 2 \mathrm{H}, \mathrm{CH}_{2}\right), 3.97\left(\mathrm{~s}, 3 \mathrm{H}, \mathrm{OCH}_{3}\right) .{ }^{13} \mathrm{C}$ NMR $(125 \mathrm{MHz}$, 
$\left.\mathrm{CDCl}_{3}\right): 176.1,175.1,165.7,159.0,143.5,142.3,141.4,135.1,134.3,129.5,127.2,126.7$, 125.7, 124.5, 123.1, 122.5, 119.6, 116.9, 114.7, 55.4, 43.0. MS m/z (\%) 419 ([M + 2], 21), 417 (M+, 69), 242 (100), 228 (24), 211 (25), 208 (30), 152 (28), 91 (24), 65 (12), 43 (12). Anal. Calcd for $\mathrm{C}_{23} \mathrm{H}_{16} \mathrm{ClN}_{3} \mathrm{O}_{3}: \mathrm{C}, 66.11 ; \mathrm{H}, 3.86 ; \mathrm{N}, 10.06$. Found: C, 65.98; H, 4.03; N, 10.29.

5.4.13. 2-Methoxy-10-[3-(4-methoxyphenyl)-[1,2,4]oxadiazol-5-ylmethyl]-10H-acridin-9-one (11m). Yellow crystals; yield: $73 \%, \mathrm{mp} 232-233{ }^{\circ} \mathrm{C} . \mathrm{IR}(\mathrm{KBr}): 3140,2927,1635,1593,1485$, $1464 \mathrm{~cm}^{-1} .{ }^{1} \mathrm{H}$ NMR $\left(500 \mathrm{MHz}, \mathrm{CDCl}_{3}\right): 8.63\left(\mathrm{dd}, J=8.0,1.3 \mathrm{~Hz}, 1 \mathrm{H}, \mathrm{H}_{8}\right), 8.03-8.01(\mathrm{~m}, 3 \mathrm{H}$, $\left.\mathrm{H}_{1}, \mathrm{H}_{4}, \mathrm{H}_{5}\right), 7.80-7.77\left(\mathrm{~m}, 1 \mathrm{H}, \mathrm{H}_{3}\right), 7.64\left(\mathrm{~d}, J=8.3 \mathrm{~Hz}, 2 \mathrm{H}, \mathrm{H}_{2}, \mathrm{H}_{6}\right), 7.43(\mathrm{dd}, J=9.2,3.1 \mathrm{~Hz}$, $\left.1 \mathrm{H}, \mathrm{H}_{6}\right), 7.39\left(\mathrm{t}, J=7.4 \mathrm{~Hz}, 1 \mathrm{H}, \mathrm{H}_{7}\right), 7.00\left(\mathrm{~d}, J=8.3 \mathrm{~Hz}, 2 \mathrm{H}, \mathrm{H}_{3^{\prime}}, \mathrm{H}_{5^{\prime}}\right), 5.70\left(\mathrm{~s}, 2 \mathrm{H}, \mathrm{CH}_{2}\right), 3.99(\mathrm{~s}$, $\left.3 \mathrm{H}, \mathrm{OCH}_{3}\right), 3.90\left(\mathrm{~s}, 3 \mathrm{H}, \mathrm{OCH}_{3}\right) .{ }^{13} \mathrm{C} \mathrm{NMR}\left(125 \mathrm{MHz}, \mathrm{CDCl}_{3}\right): 176.0,175.0,165.7,159.0$, 154.3, 142.3, 141.4, 136.8, 133.3, 127.5, 126.7, 125.0, 123.0, 122.3, 120.4, 118.6, 116.7, 114.1, 106.0, 55.8, 55.3, 43.0. MS m/z (\%) 413 (M+, 70), 238 (100), 205 (22), 187 (31), $152(31), 129$ (61), 91 (35), 65 (8), 43 (14). Anal. Calcd for $\mathrm{C}_{24} \mathrm{H}_{19} \mathrm{~N}_{3} \mathrm{O}_{4}$ : C, 69.72; H, 4.63; N, 10.16. Found: C, 69.56; H, 4.84; N, 10.33 .

5.4.14.2-Bromo-10-[3-(4-methoxyphenyl)-[1,2,4]oxadiazol-5-ylmethyl]-10H-acridin-9-one (11n). White crystals; yield: $64 \%, \mathrm{mp}>250^{\circ} \mathrm{C}$. IR (KBr): 3054, 2924, 1635, 1595, 1484, 1458 $\mathrm{cm}^{-1} .{ }^{1} \mathrm{H}$ NMR $\left(500 \mathrm{MHz}, \mathrm{CDCl}_{3}\right): 8.73\left(\mathrm{~d}, J=2.1 \mathrm{~Hz}, 1 \mathrm{H}, \mathrm{H}_{1}\right), 8.61\left(\mathrm{~d}, J=7.9 \mathrm{~Hz}, 1 \mathrm{H}, \mathrm{H}_{8}\right)$, $8.01\left(\mathrm{~d}, J=8.5 \mathrm{~Hz}, 2 \mathrm{H}, \mathrm{H}_{4}, \mathrm{H}_{5}\right), 7.87\left(\mathrm{dd}, J=9.10,2.5 \mathrm{~Hz}, 1 \mathrm{H}, \mathrm{H}_{6}\right), 7.84-7.81\left(\mathrm{~m}, 1 \mathrm{H}, \mathrm{H}_{3}\right), 7.65$ $\left(\mathrm{d}, J=8.5 \mathrm{~Hz}, 2 \mathrm{H}, \mathrm{H}_{2^{\prime}}, \mathrm{H}_{6^{\prime}}\right), 7.41\left(\mathrm{t}, J=7.5 \mathrm{~Hz}, 1 \mathrm{H}, \mathrm{H}_{7}\right), 7.00\left(\mathrm{~d}, J=8.5 \mathrm{~Hz}, 2 \mathrm{H}, \mathrm{H}_{3^{\prime}}, \mathrm{H}_{5^{\prime}}\right), 5.76$ (s, $\left.2 \mathrm{H}, \mathrm{CH}_{2}\right), 3.90\left(\mathrm{~s}, 3 \mathrm{H}, \mathrm{OCH}_{3}\right) .{ }^{13} \mathrm{C} \mathrm{NMR}\left(125 \mathrm{MHz}, \mathrm{DMSO}-d_{6}\right): 175.6,174.2,162.5,159.1$, $145.8,142.1,141.3,136.3,134.4,128.9,128.4,127.0,126.6,123.0,122.0,121.6,119.4,116.5$, 114.0, 55.1, 42.8. Anal. Calcd for $\mathrm{C}_{23} \mathrm{H}_{16} \mathrm{BrN}_{3} \mathrm{O}_{3}:$ C, 59.76; H, 3.49; N, 9.09. Found: C, 59.37; H, 3.81; N, 9.32. 


\subsection{Anticonvulsant activity}

\subsubsection{Animals and drugs}

Male mice (Pasteur Institute, Iran), weighing 20-25 g, were used as experimental animals. Mice were housed in a temperature-controlled condition $\left(25-30^{\circ} \mathrm{C}\right)$ and $12 \mathrm{~h}$ light/dark cycle. They were allowed to acclimatize with free access to food and water for a $24 \mathrm{~h}$ period before testing except during the experiment. Animals were arranged to experimental groups randomly and each mouse was used only one time for the experiments. Phenobarbital (Sigma) and diazepam (Sigma) were used as a reference drug for comparison. Pentylenetetrazole (PTZ, Sigma) was used to induce convulsions in the experimental animals.

\subsubsection{Pentylenetetrazole (PTZ)-induced seizures test}

The anticonvulsant activity of the novel compounds was determined with PTZ-induced seizure test. In this model of induced seizure, the ability of the novel compounds to protect mice against lethal dose of PTZ ( $80 \mathrm{mg} / \mathrm{kg}$ ) was evaluated. The test compounds were dissolved in 5\% DMSO (in saline) and injected intraperitoneally (i.p.) in the doses of 10, 5, 2.5, $1 \mathrm{mg} / \mathrm{kg}$ animal weigh using the same dosing volume of $0.2 \mathrm{ml}$ per $20 \mathrm{~g}$. PTZ was dissolved in normal saline in $2 \%$ concentration and was given i.p. in a dose of $80 \mathrm{mg} / \mathrm{kg}$ body weight. Phenobarbital and diazepam were dissolved in normal saline in $2 \%$ concentration and they were i.p. given in the doses of 10 , $5,2.5$, and $1 \mathrm{mg} / \mathrm{kg}$ for phenobarbital and $1,0.5$, and $0.25 \mathrm{mg} / \mathrm{kg}$ for diazepam using the same dosing volume. All drugs were freshly prepared to the desired concentration just before use. Control animals received an equal volume of vehicle (5\% DMSO in saline). After 30 min, PTZ was injected i.p. to animals. Observations were made at least $30 \mathrm{~min}$ after the administration of PTZ and the number of dead mice was recorded. The median effective dose $\left(\mathrm{ED}_{50}\right)$ was 
calculated by probit-regression method and SPSS software (Chicago, IL; version 13). The ED 50 values were presented as mean with $95 \%$ confidence intervals calculated by probit analysis. $P$ $<0.05$ was considered as statistically significant.

In addition, the selected compound 11l, vehicle, or diazepam, as reference BZD agonist, were administered $30 \mathrm{~min}$ and flumazenil, as antagonist of BZD receptors, $15 \mathrm{~min}$ before the experiments. Then PTZ (80 mg/kg; i.p.) was injected. Mice were under observation for $30 \mathrm{~min}$ after the injection of PTZ and the dead mice were counted.

\subsubsection{Maximal electroshock (MES) induced seizures test}

The ability of the novel compounds 11a-n to prevent MES-induced seizures was evaluated in mice. The test compounds were dissolved in 5\% DMSO (in saline) and injected intraperitoneally (i.p.) in the doses of $10,5,2.5,1 \mathrm{mg} / \mathrm{kg}$ animal weigh using the same dosing volume of $0.2 \mathrm{ml}$ per 20 g. Phenobarbital and diazepam (i.p.) were used as standard drugs. Phenobarbital was given at the doses of $1,2.5,5$, and $10 \mathrm{mg} / \mathrm{kg}$. Diazepam was administrated at the doses of 0.25 , 0.5 , and $1 \mathrm{mg} / \mathrm{kg}$. Control animals received an equal volume of vehicle (5\% DMSO in saline). The occurrence of hind limb tonic extension (HLTE) in mice following applying MES (60 Hz, $37.2 \mathrm{~mA}$ and $0.25 \mathrm{~s}, 30 \mathrm{~min}$ after intraperitoneal administration of the tested compounds) was assessed. The electrical current was applied through ear electrodes and mice were observed for $30 \mathrm{~s}$ for incidence of HLTE. The results were recorded as number of animal protected/number of animals tested. The $\mathrm{ED}_{50}$ was calculated by probit-regression method and SPSS software (Chicago, IL; version 13). The $\mathrm{ED}_{50}$ values were presented as mean with $95 \%$ confidence intervals calculated by probit analysis. $P<0.05$ was considered as statistically significant.

\subsection{Neurotoxicity screening}


The neurotoxicity of compounds 11a-n was studied by evaluating minimal motor impairment in mice using rotarod test. The animals were placed on a 1 inch diameter knurled plastic rod rotating at $6 \mathrm{rpm}$. Trained animals were treated with the test compounds (i.p. injection) at the doses of 30,100 , and $300 \mathrm{mg} / \mathrm{kg}$. Neurotoxicity was indicated by the inability of the animal to maintain equilibrium on the rod for at least $1 \mathrm{~min}$ in each of the four trials [19]. The neurotoxicity was expressed as the median toxic dose $\left(\mathrm{TD}_{50}\right.$ in $\mathrm{mg} / \mathrm{kg}$ ) eliciting minimal neurological toxicity in $50 \%$ of animals.

\subsection{Cytotoxicity screening}

The human cancer cell lines MCF-7, T-47D and MDA-MB-231 were purchased from the National Cell Bank of Iran (NCBI). The cells were cultured in RPMI 1640 medium supplemented with $10 \%$ heat-inactivated fetal calf serum and $100 \mu \mathrm{g} / \mathrm{mL}$ streptomycin and 100 $\mathrm{U} / \mathrm{mL}$ penicillin at $37^{\circ} \mathrm{C}$ in $5 \% \mathrm{CO}_{2}$-humidified atmosphere. The cells $\left(5 \times 10^{4}\right.$ cells $/ \mathrm{mL}$ in 96 well culture plates) were incubated for $48 \mathrm{~h}$ with different concentrations of compounds 11a-n dissolved in DMSO. Etoposide and DMSO were used as positive and negative controls, respectively. The viability of cells was determined by using MTT method [20]. All experiments were performed three times and the $\mathrm{IC}_{50}$ values for all compounds were calculated by nonlinear regression analysis.

\subsection{Molecular modeling}

The homology model of the diazepam-bound $\mathrm{GABA}_{\mathrm{A}}$ receptor developed by Ernst et al. was retrieved from the Supplementary material of their published paper [14]. The 3D structure of compound 11 was provided using MarvineSketch 5.8.3, 2012, ChemAxon (http:// www.chemaxon.com) and converted to pdbqt format file using AutoDockTools version1.5.6. 
(http://mgltools.scripps.edu). Also, the autodock format of protein was provided using the same software. Polar hydrogens and Kollman united atom partial charges were assigned to the individual protein atoms. A docking grid box was built with 40,40 and 40 points in $\mathrm{x}=43.640$, $\mathrm{y}$ $=43.866$ and $\mathrm{z}=9.3290$ directions and each docked system was carried out by 100 runs of the AUTODOCK search by the Lamarckian genetic algorithm (LGA). The lowest energy conformation of ligand-enzyme complex was evaluated by Discovery Studio 4.0 Client.

\section{Acknowledgment}

This work was supported by grants from the Research Council of Tehran University of Medical Sciences (grant no. 94-02-45-29071) and Iran National Science Foundation (INSF).

\section{References}

[1] W. Loscher, New visions in the pharmacology of anticonvulsion, Eur. J. Pharmacol. 342 (1998) 1-13.

[2] A.C. Swann, Major system toxicities and side effects of anticonvulsants, J. Clin. Psychiatry. $62(2001) 16-21$.

[3] P. Kwan, and M.J. Brodie, Early identification of refractory epilepsy, N. Engl. J. Med. 342 (2000) 314-319.

[4] B.B. Spear, Pharmacogenetics and antiepileptic drugs, Epilepsia. 42 (2001) 31-34.

[5] (a) N. Ahangar, A. Ayati, E. Alipour, A. Pashapour, A. Foroumadi, S. Emami, 1-[(2Arylthiazol-4-yl)methyl]azoles as a new class of anticonvulsants: design, synthesis, in vivo screening, and in silico drug-like properties. Chem. Biol. Drug Des. 78 (2011) 844-852. 
(b) A. Almasirad, S.A. Tabatabai, M. Faizi, A. Kebriaeezadeh, N. Mehrabi, A. Dalvandi, and A. Shafiee, Synthesis and anticonvulsant activity of new 2-substituted-5-[2-(2fluorophenoxy) phenyl]-1,3,4-oxadiazoles and 1,2,4-triazoles, Bioorg. Med. Chem. Lett. 14 (2004) 6057-6059.

[6] H.J. Lankau, K. Unverferth, C. Grunwald, H. Hartenhauer, K. Heinecke, K. Bernöster, R. Dost, U. Egerland, C. Rundfeldt, New GABA-modulating 1,2,4-oxadiazole derivatives and their anticonvulsant activity, Eur. J. Med. Chem. 42 (2007) 873-879.

[7] S.E. Mayer, J.A. Bain, The distribution of a new central convulsant: 10-(2dimethylaminopropyl)-9-acridone. J. Pharmacol. Exp. Ther. 111 (1954) 210-223.

[8] M. Mohammadi-Khanaposhtani, M. Saeedi, M. Mahdavi, N.S. Zafarghandi, R. Sabourian, E.K. Razkenari, H. Alinezhad, M. Khanavi, A. Foroumadi, A. Shafiee, T. Akbarzadeh, Potent acetylcholinesterase inhibitors: Design, synthesis, biological evaluation, and docking study of acridone linked to 1,2,3-triazole derivatives, Eur. J. Med. Chem. 92 (2015) 799806.

[9] M. Faizi, S. Dabirian, H. Tajali, F. Ahmadi, E.R. Zavareh, S. Shahhosseini, S.A. Tabatabai, Novel agonists of benzodiazepine receptors: Design, synthesis, binding assay and pharmacological evaluation of 1,2,4-triazolo [1,5-a] pyrimidinone and 3-amino-1,2,4triazole derivatives, Bioorg. Med. Chem. 23 (2015) 480-487.

[10] C. Wolf, S. Liu, X. Mei, A.T. August, M. Casimir, Regioselective copper-catalyzed amination of bromobenzoic acids using aliphatic and aromatic amines, J. Org. Chem. 71 (2006) 3270-3273. 
[11] R. Hedge, P. Thimmaiah, M.C. Yerigeri, G. Krishnegowda, K.N. Thimmaiah, P.J. Houghton, Anti-calmodulin acridone derivatives modulate vinblastine resistance in multidrug resistant (MDR) cancer cells, Eur. J. Med. Chem. 39 (2004) 161-178.

[12] D. Kumar, G. Patel, E.O. Johnson, K. Shah, Synthesis and anticancer activities of novel 3,5disubstituted-1,2,4-oxadiazoles, Bioorg. Med. Chem. Lett. 19 (2009) 2739-2741.

[13] (a) W. Löscher, Critical review of current animal models of seizures and epilepsy used in the discovery and development of new antiepileptic drugs. Seizure 20 (2011) 359-368.

(b) W. Löscher, D. Hönack, C.P. Fassbender, B. Nolting, The role of technical, biological and pharmacological factors in the laboratory evaluation of anticonvulsant drugs. III. Pentylenetetrazole seizure models, Epilepsy Res. 8 (1991) 171-189.

[14] L. Richter, C. de Graaf, W. Sieghart, Z. Varagic, M. Mörzinger, I.J. de Esch, G.F Ecker, M. Ernst, Diazepam-bound $\mathrm{GABA}_{\mathrm{A}}$ receptor models identify new benzodiazepine binding-site ligands. Nat. Chem. Biol. 8 (2012) 455-464.

[15] G. Cholewiński, K. Dzierzbicka, A.M. Kołodziejczyk, Natural and synthetic acridines/acridones as antitumor agents: their biological activities and methods of synthesis. Pharmacol. Rep. 63 (2011) 305-336.

[16] A. George, The design and molecular modeling of CNS drugs. Curr. Opin. Drug Discov. Dev. 2 (1999) 286-292.

[17] M.H. Abraham, K. Takács-Novák, R.C. Mitchell, On the partition of ampholytes: application to blood-brain distribution. J. Pharm. Sci. 86 (1997) 310-315.

[18] D.E. Clark, Rapid calculation of polar molecular surface area and its application to the prediction of transport phenomena. 2. Prediction of blood-brain barrier penetration. J. Pharm. Sci. 88 (1999) 815-821. 
[19] N.W. Dunham, T.A. Miya, L.D. Edwards, The pharmacological activity of a series of basic esters of mono- and dialkylmalonic acids, J. Am. Pharm. Assoc. 46 (1957) 64-66.

[20] T. Mosmann, Rapid colorimetric assay for cellular growth and survival: application to proliferation and cytotoxicity assays. J. Immunol. Methods 65 (1983) 55-63. 


\section{Captions:}

Figure 1. The structures of lead compounds I and II, and designed compounds 11a-n.

Figure 2. Pharmacophoric features of benzodiazepine agonists (Diazepam and Estazolam), and the new designed compounds 11a-n.

Figure 3. (a) Diazepam and (b) the most active compound $\mathbf{1 1}$ in the BZD-binding pocket of $\mathrm{GABA}_{\mathrm{A}}$.

Scheme 1. Synthesis of compounds 11a-n. 


\section{Table 1}

Anticonvulsant activity of compounds 11a-n in PTZ-induced convulsion test.<smiles>[R]c1ccc(-c2noc(Cn3c4ccccc4c(=O)c4cc([R])ccc43)n2)cc1</smiles>

\begin{tabular}{|c|c|c|c|c|c|c|c|}
\hline \multirow[t]{2}{*}{ Compound } & \multirow[t]{2}{*}{$\mathbf{R}_{1}$} & \multirow[t]{2}{*}{$\mathbf{R}_{2}$} & \multicolumn{2}{|c|}{$E D_{50}{ }^{a}(\mathrm{mg} / \mathrm{kg})$} & \multirow[t]{2}{*}{$\mathrm{TD}_{50}(\mathrm{mg} / \mathrm{kg})$} & \multicolumn{2}{|c|}{$\overline{\mathbf{P I}^{\mathrm{c}}}$} \\
\hline & & & PTZ & MES & & PTZ & MES \\
\hline 11a & $\mathrm{H}$ & $\mathrm{Me}$ & $\begin{array}{l}6.37 \\
(4.43-10.24)^{b}\end{array}$ & $\begin{array}{l}2.52 \\
(1.88-3.06)\end{array}$ & $\begin{array}{l}538.3 \\
(488.4-594.7)\end{array}$ & 84.5 & 213.6 \\
\hline $11 b$ & $\mathrm{Cl}$ & $\mathrm{Me}$ & $\begin{array}{l}6.25 \\
(4.68-9.52)\end{array}$ & $\begin{array}{l}2.24 \\
(1.74-2.93)\end{array}$ & $\begin{array}{l}483.2 \\
(437.1-532.6)\end{array}$ & 77.3 & 215.7 \\
\hline $11 \mathrm{c}$ & $\mathrm{OMe}$ & $\mathrm{Me}$ & $\begin{array}{l}6.32 \\
(4.98-11.02)\end{array}$ & $\begin{array}{l}3.25 \\
(2.35-4.00)\end{array}$ & $\begin{array}{l}521.8 \\
(473.3-572.5)\end{array}$ & 82.4 & 160.5 \\
\hline 11d & $\mathrm{Br}$ & $\mathrm{Me}$ & $>50$ & $\begin{array}{l}5.12 \\
(4.16-7.01)\end{array}$ & $\begin{array}{l}594.5 \\
(538.1-649.3)\end{array}$ & $<11.9$ & 116.1 \\
\hline $11 \mathrm{e}$ & $\mathrm{Me}$ & $\mathrm{Me}$ & $>50$ & $\begin{array}{l}2.77 \\
(2.18-3.28)\end{array}$ & $\begin{array}{l}542.3 \\
(488.7-601.2)\end{array}$ & $<10.8$ & 195.8 \\
\hline $11 f$ & Et & $\mathrm{Me}$ & $\begin{array}{l}18.93 \\
(10.62-25.31)\end{array}$ & $\begin{array}{l}8.31 \\
(6.21-10.51)\end{array}$ & $\begin{array}{l}495.7 \\
(447.6-546.8)\end{array}$ & 26.1 & 59.6 \\
\hline $11 \mathrm{~g}$ & $\mathrm{H}$ & $\mathrm{Cl}$ & $\begin{array}{l}5.78 \\
(3.53-8.12)\end{array}$ & $\begin{array}{l}3.57 \\
(1.61-4.38)\end{array}$ & $\begin{array}{l}528.2 \\
(475.2-580.4)\end{array}$ & 91.3 & 147.9 \\
\hline $11 \mathrm{~h}$ & $\mathrm{Cl}$ & $\mathrm{Cl}$ & $\begin{array}{l}4.16 \\
(2.51-7.33)\end{array}$ & $\begin{array}{l}2.64 \\
(1.75-3.24)\end{array}$ & $\begin{array}{l}454.5 \\
(401.5-513.3)\end{array}$ & 109.1 & 172.1 \\
\hline 11i & $\mathrm{OMe}$ & $\mathrm{Cl}$ & $\begin{array}{l}5.22 \\
(3.47-9.42)\end{array}$ & $\begin{array}{l}3.68 \\
(3.18-4.19)\end{array}$ & $\begin{array}{l}552.6 \\
(498.9-611.2)\end{array}$ & 105.7 & 150.2 \\
\hline $11 \mathrm{j}$ & $\mathrm{Br}$ & $\mathrm{Cl}$ & $\begin{array}{l}7.57 \\
(5.73-13.56)\end{array}$ & $\begin{array}{l}2.87 \\
(2.30-3.40)\end{array}$ & $\begin{array}{l}512.1 \\
(461.3-563.6)\end{array}$ & 67.6 & 178.4 \\
\hline $11 k$ & $\mathrm{Me}$ & $\mathrm{Cl}$ & $\begin{array}{l}15.15 \\
(7.71-19.19)\end{array}$ & $\begin{array}{l}6.53 \\
(4.41-7.52)\end{array}$ & $\begin{array}{l}474.8 \\
(427.7-526.2)\end{array}$ & 31.3 & 72.7 \\
\hline 111 & $\mathrm{Cl}$ & $\mathrm{OMe}$ & $\begin{array}{l}2.08 \\
(1.35-3.41)\end{array}$ & $\begin{array}{l}3.71 \\
(2.76-4.34)\end{array}$ & $\begin{array}{l}438.3 \\
(392.9-490.5)\end{array}$ & 210.6 & 118.1 \\
\hline $11 \mathrm{~m}$ & $\mathrm{OMe}$ & $\mathrm{OMe}$ & $\begin{array}{l}2.12 \\
(1.81-3.74)\end{array}$ & $\begin{array}{l}2.42 \\
(1.82-2.90)\end{array}$ & $\begin{array}{l}461.7 \\
(419.1-507.3)\end{array}$ & 217.4 & 190.8 \\
\hline $11 n$ & $\mathrm{Br}$ & $\mathrm{OMe}$ & $\begin{array}{l}6.21 \\
(4.61-11.53)\end{array}$ & $\begin{array}{l}8.71 \\
(6.16-10.13)\end{array}$ & $\begin{array}{l}471.2 \\
(426.5-522.8)\end{array}$ & 75.8 & 54.1 \\
\hline Phenobarbital & - & - & $\begin{array}{l}12.45 \\
(8.73-16.11)\end{array}$ & $\begin{array}{l}20.6 \\
(18.8-24.3)\end{array}$ & $\begin{array}{l}68.9 \\
(64.4-73.1)\end{array}$ & 5.5 & 3.3 \\
\hline Diazepam & - & - & $\begin{array}{l}0.68 \\
(0.41-0.83)\end{array}$ & $\begin{array}{l}0.98 \\
(0.69-1.22)\end{array}$ & $\mathrm{nd}^{\mathrm{d}}$ & nd & nd \\
\hline
\end{tabular}

${ }^{\mathrm{a}} \mathrm{ED}_{50}$ median effective dose affording anticonvulsant protection in $50 \%$ of animals

${ }^{\mathrm{b}} 95 \%$ confidence intervals given in parentheses

${ }^{c} \mathrm{PI}=$ Protective Index $\left(\mathrm{TD}_{50} / \mathrm{ED}_{50}\right)$

${ }^{\mathrm{d}}$ Not determind 

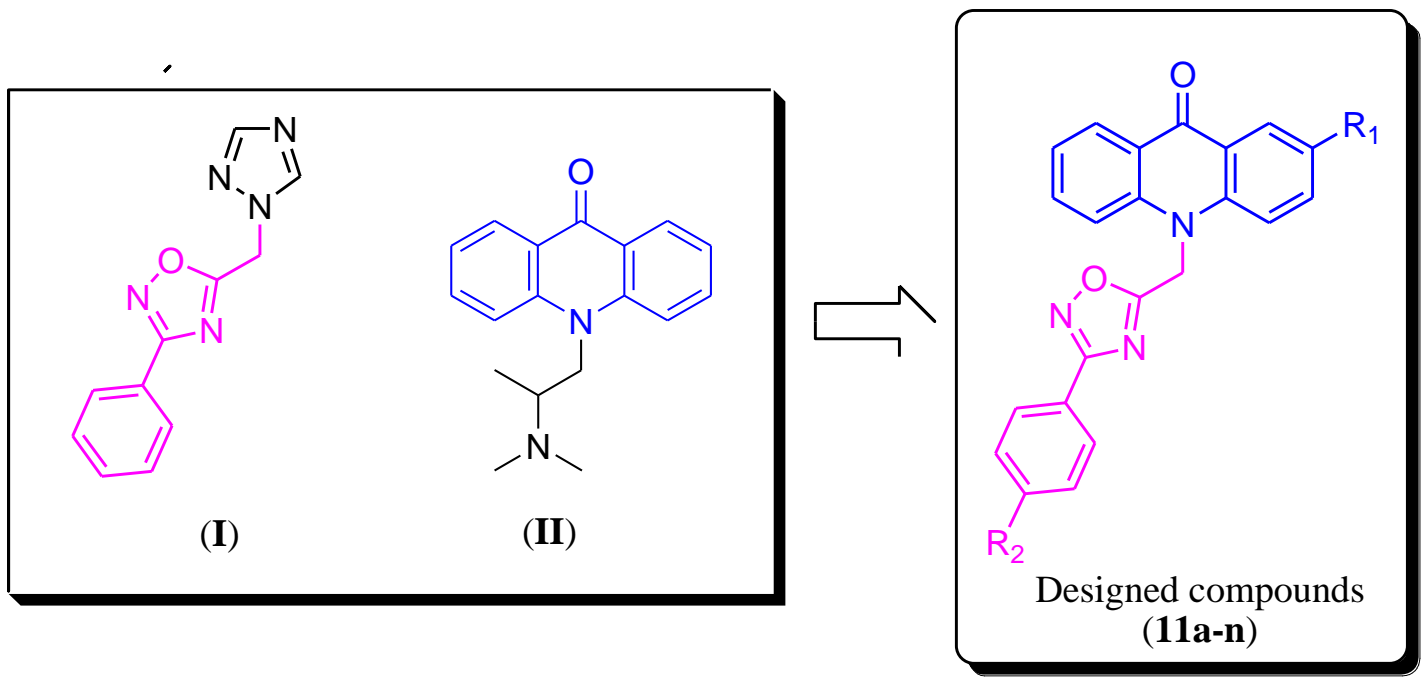

Figure 1. The structures of lead compounds I and II, and designed compounds 11a-n. 


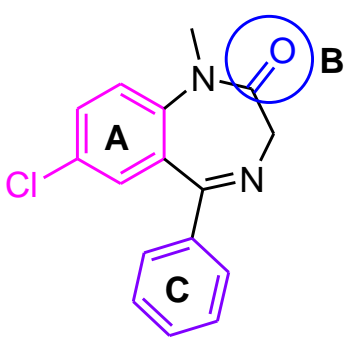

Diazepam

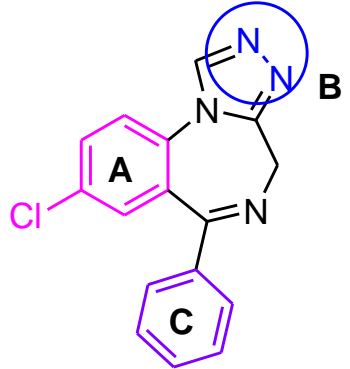

Estazolam

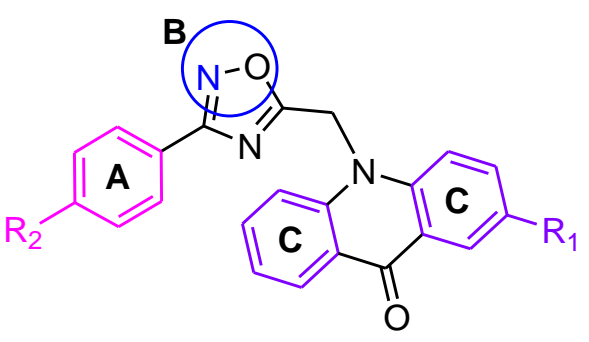

Designed compounds
A) aromatic ring
B) coplanar proton-accepting group
C) out-of-plane aromatic ring

Figure 2. Pharmacophoric features of benzodiazepine agonists (Diazepam and Estazolam), and the new designed compounds 11a-n. 


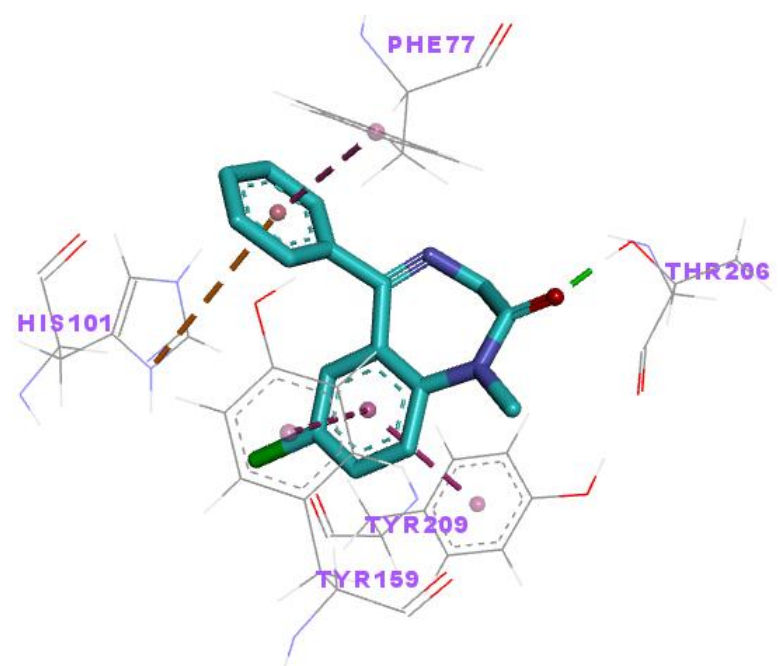

(a)

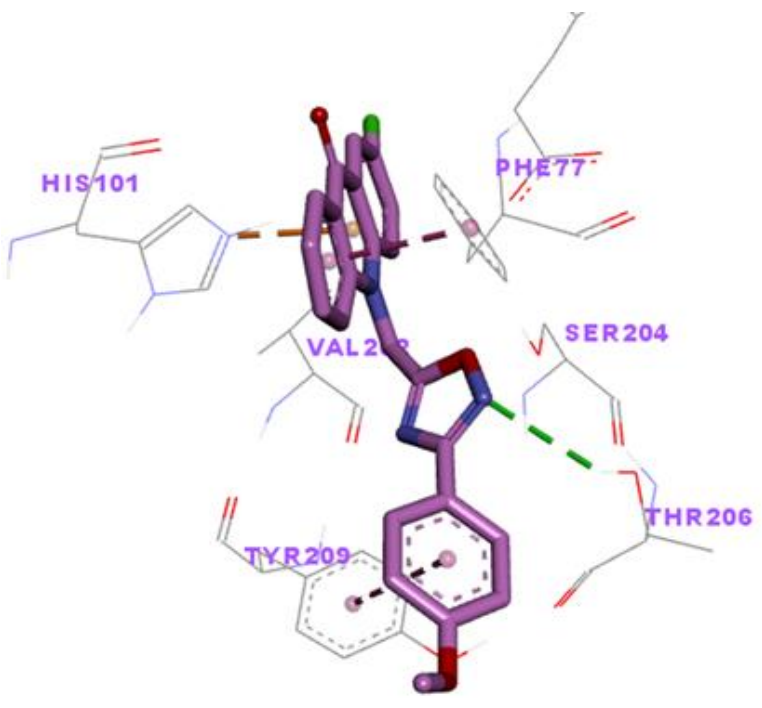

(b)

Figure 3. (a) Diazepam and (b) the most active compound $\mathbf{1 1 l}$ in the BZD-binding pocket of $\mathrm{GABA}_{\mathrm{A}}$. 


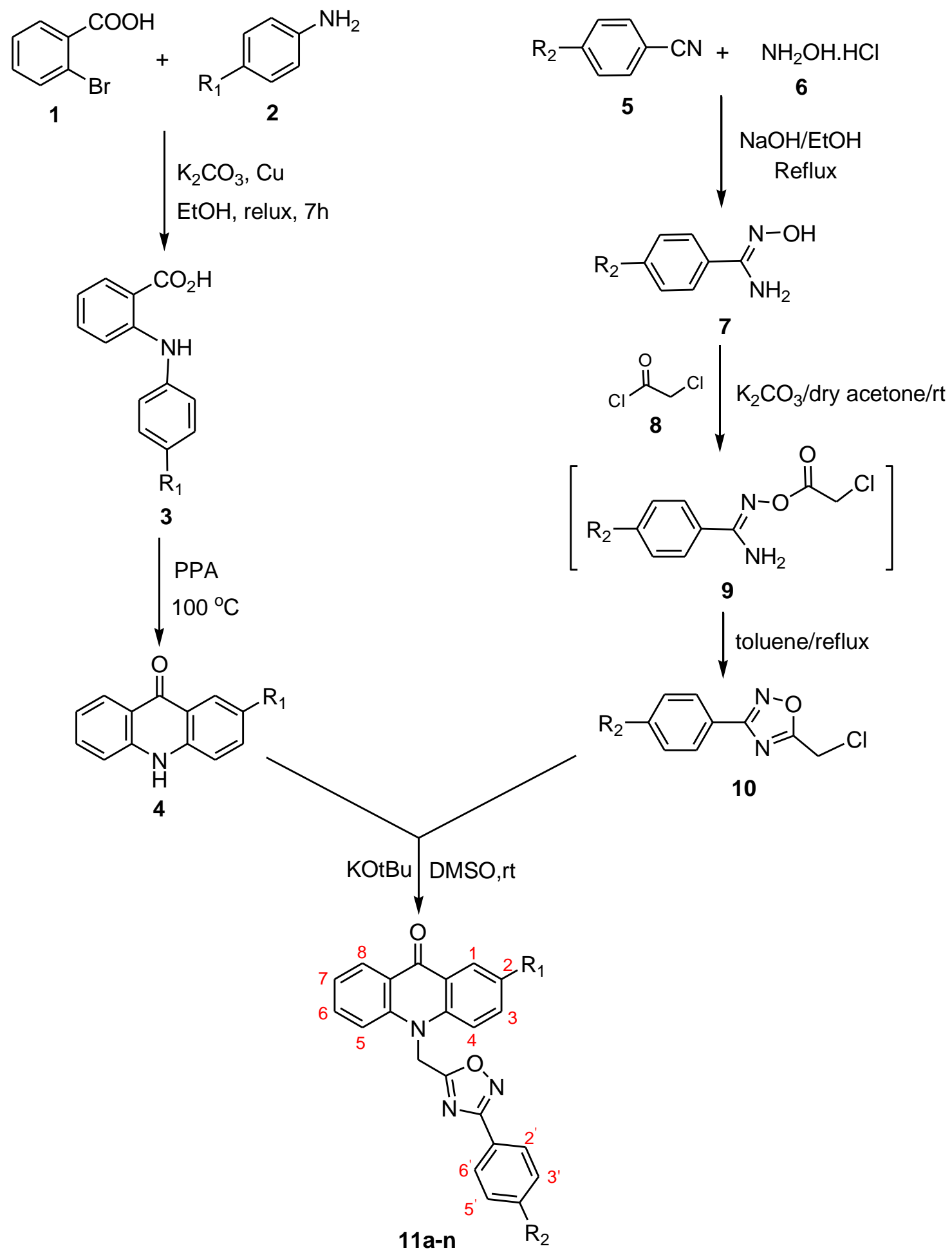

Scheme 1. Synthesis of compounds 11a-n. 


\section{Graphical Abstract}

Design, synthesis, pharmacological evaluation, and docking study of new acridonebased 1,2,4-oxadiazoles as potential anticonvulsant agents

Maryam Mohammadi-Khanaposhtani, Mohammad Shabani, Mehrdad Faizi, Iraj Aghaei, Reza Jahani, Zainab Sharafi, Narges Shamsaei Zafarghandi, Mohammad Mahdavi, Tahmineh Akbarzadeh, Saeed Emami, Abbas Shafiee, Alireza Foroumadi *
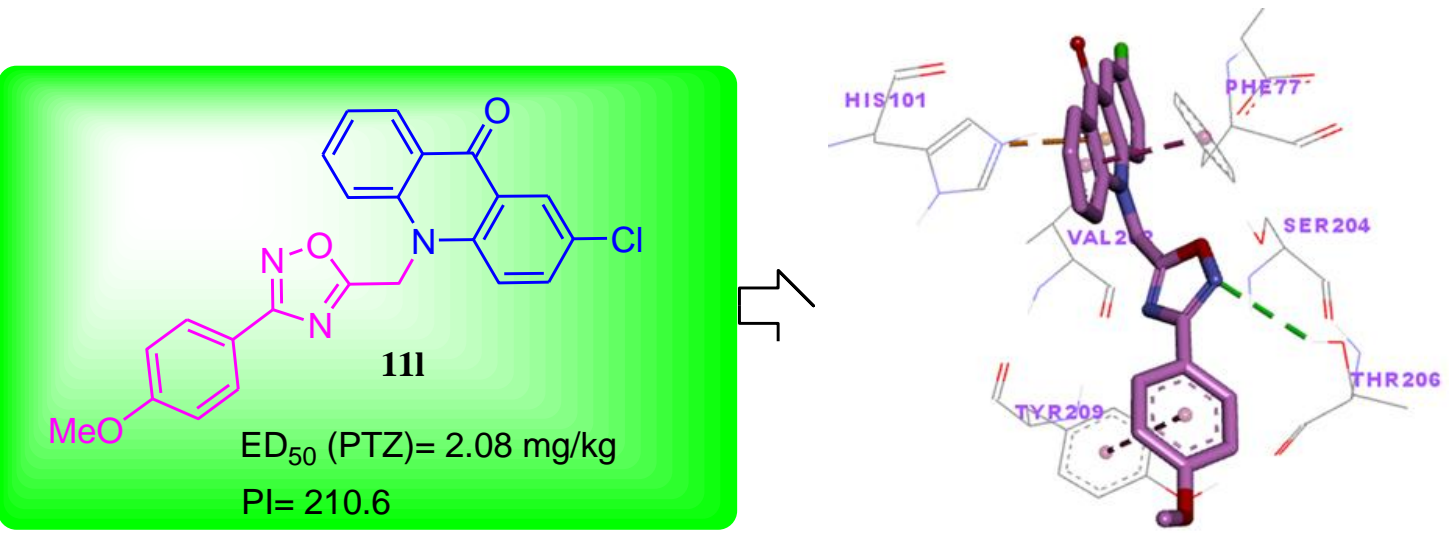

A series of acridone-based oxadiazoles were synthesized and evaluated as new anticonvulsant agents. The 2-chloro-acridinone derivative $\mathbf{1 1 l}$ was the most potent compound in the PTZ test. 